\title{
Large enhancement of the effective second-order nonlinearity in graphene metasurfaces
}

\author{
Qun Ren, J. W. You, and N. C. Panoiu \\ Department of Electronic and Electrical Engineering, University College London, Torrington Place, London WC1E 7JE, United Kingdom
}

(Received 30 January 2019; revised manuscript received 12 April 2019; published 6 May 2019)

\begin{abstract}
Using a powerful homogenization technique, one- and two-dimensional graphene metasurfaces are homogenized both at the fundamental frequency (FF) and second harmonic ( $\mathrm{SH}$ ). In both cases, there is excellent agreement between the predictions of the homogenization method and those based on rigorous numerical solutions of Maxwell equations. The homogenization technique is then employed to demonstrate that, owing to a double-resonant plasmon excitation mechanism that leads to strong, simultaneous field enhancement at the FF and SH, the effective second-order susceptibility of graphene metasurfaces can be enhanced by more than three orders of magnitude as compared to the intrinsic second-order susceptibility of a graphene sheet placed on the same substrate. In addition, we explore the implications of our results on the development of new active nanodevices that incorporate nanopatterned graphene structures.
\end{abstract}

DOI: 10.1103/PhysRevB.99.205404

\section{INTRODUCTION}

Metamaterials, which consist of artificial elements (socalled metaatoms or metamolecules) usually arranged in a periodic pattern, have been playing an increasingly important role in applications in which they emulate physical properties that otherwise cannot be achieved with naturally occurring materials. The broad available choice of particular geometries and material parameters of the constituents of metamaterials facilitates their use for the implementation of key functionalities, including, inter alia, phase engineering [1-3], light focusing [4-6], and local field enhancement [7-10]. These functionalities are beginning to impact a series of research fields by finding applications to bio-sensing [11-14], development of efficient absorbers [15-17], electromagnetic cloaking $[18,19]$, and imaging beyond subdiffraction limits [20-23]. Among these physical properties of metamaterials, local field enhancement is particularly relevant to nonlinear optics, since in this case the optical response of a metamaterial-based device depends nonlinearly on the externally applied optical field and thus can be widely tuned.

In many applications, the two-dimensional (2D) counterpart of metamaterials, the so-called metasurfaces, can provide the required functionality, especially in the case of devices with planar configuration. In addition, metasurfaces have the advantage of requiring much less laborious fabrication processes. Moreover, in many applications pertaining to nonlinear optics, especially those related to surface science and sensing, achieving the phase-matching of the interacting waves is not a prerequisite condition, and therefore the constraints imposed on metasurfaces in order to attain optimal energy conversion in nonlinear processes can be greatly relaxed [24-28].

Broadly speaking, there are two classes of optical metasurfaces: plasmonic metasurfaces based on metallic particles $[29,30]$ and dielectric metasurfaces [31,32] relying on Mie resonances of dielectric particles. In the case of plasmonic metasurfaces, the local field can be dramatically enhanced at plasmon-resonance frequencies [33-36]; however, this effect is usually accompanied by a relatively large optical loss [37]. On the other hand, dielectric metasurfaces are characterized by much smaller optical losses but usually provide reduced optical field enhancement.

A promising alternative to plasmonic and all-dielectric metasurfaces is provided by graphene metasurfaces, as the (plasmon) resonance frequency of graphene nanostructures lies in the terahertz domain, namely, where optical losses of graphene are relatively small. Equally important, the plasmonic nature of these resonances ensures that strong field enhancement can be achieved in graphene metasurfaces, too. In addition, the corresponding resonance wavelength is much larger than the size of graphene resonators, which means that a large number of such resonators can be packed inside a domain with size comparable to that of the operating wavelength. Consequently, the optical response of graphene metasurfaces can be highly isotropic, when the geometry of the graphene unit cell is symmetric. In fact, patterned graphene has already been employed in the design of terahertz devices, such as perfect absorbers, filters, and tunable reflectors [38-43]. In this context, a particularly appealing physical property of graphene is the tunability of its dielectric constant, a unique functionality that is highly relevant to the design of active photonic devices.

In this paper, we propose a powerful and versatile homogenization approach for graphene metasurfaces, and subsequently use it to demonstrate that the effective secondorder susceptibility of such metasurfaces can be dramatically increased due to the field-enhancement effect at plasmon resonances. The novelty of the homogenization method used in this study consists in its ability to describe not only metasurfaces containing linear and isotropic materials, such as the standard field-average methods, but also those made of anisotropic and nonlinear optical media. In addition, we find that when a so-called double-resonance phenomenon occurs in a graphene metasurface [44], the second-harmonic generation (SHG) can be further enhanced, leading to an overall increase in SHG of more than three orders of magnitude as 
compared to the SHG of a graphene sheet placed on the same substrate.

The paper is organized as follows: In the next section, the configurations of the graphene metasurfaces investigated in this work are described, as well as their material parameters. In Sec. III, an improved homogenization approach for retrieving the effective linear and nonlinear properties of graphene metasurfaces is presented. Then, using this homogenization method, the geometrical parameters of the graphene metasurfaces are optimized so as to achieve plasmon resonances at both the fundamental frequency (FF) and second harmonic (SH). In Sec. IV, the linear and nonlinear optical spectra of the graphene metasurfaces are calculated and a comparison of the effective second-order susceptibility of graphene metasurfaces with the second-order susceptibility of a graphene sheet placed on the same substrate is provided. Finally, the main conclusions are outlined in Sec. V.

\section{PHYSICAL CONFIGURATION AND MATERIAL PARAMETERS OF GRAPHENE METASURFACES}

In this section, we present the configuration of the onedimensional (1D) and 2D graphene metasurfaces studied in this work and describe the properties of the linear and nonlinear optical constants of graphene. Thus, the two generic nonlinear graphene-based metasurfaces, a 1D metasurface consisting of a periodic arrangement of graphene ribbons and a 2D metasurface consisting of a rectangular array of graphene rectangular patches, are schematically illustrated in Figs. 1(a) and 1(b), respectively. The period of the 1D metasurface is $P_{x}=100 \mathrm{~nm}$ and the width of the nanoribbons is $w$, whereas in the case of the $2 \mathrm{D}$ metasurface the periods along the $x$ and $y$ axes are $P_{x}=P_{y}=100 \mathrm{~nm}$ and the length of the graphene patches along the $y$ axis is fixed at $w_{y}=30 \mathrm{~nm}$. The width of the graphene nanoribbons and the length of the graphene patches along the $x$ axis, $w_{x}$, are free parameters that will be optimized so as to achieve a double-resonance effect. In both cases the graphene nanostructures are placed onto a silica substrate with $n_{\mathrm{SiO}_{2}}=1.4$ and are illuminated by a normally incident, $x$-polarized plane wave with field amplitude $E_{0}=1 \mathrm{~V} \mathrm{~m}^{-1}$ (wave intensity $I_{0}=$ $4.43 \times 10^{12} \mathrm{~W} \mathrm{~m}^{-2}$ ). This choice of the wave polarization ensures that graphene plasmons exist in both metasurfaces.

Due to its metallic characteristics in the terahertz and infrared spectral regions, graphene supports surface-plasmon polaritons (SPPs), which are collective oscillations of free electrons. In the case of finite-size graphene nanostructures, the resonance frequency of SPPs is geometry dependent. Therefore, by properly choosing the size and shape of these graphene nanostructures, one can achieve a double-resonant phenomenon, namely, SPPs exist both at the FF and $\mathrm{SH}$. When this occurs, the optical near-fields at the FF and $\mathrm{SH}$ are strongly enhanced, which leads to a marked increase of the intensity of the SHG. Under these circumstances, one expects that the graphene metasurface can be viewed as a homogeneous sheet of nonlinear material with strongly enhanced effective second-order susceptibility.

Before we analyze in more detail the linear and nonlinear optical properties of the two graphene metasurfaces, we briefly summarize the optical properties of the main optical

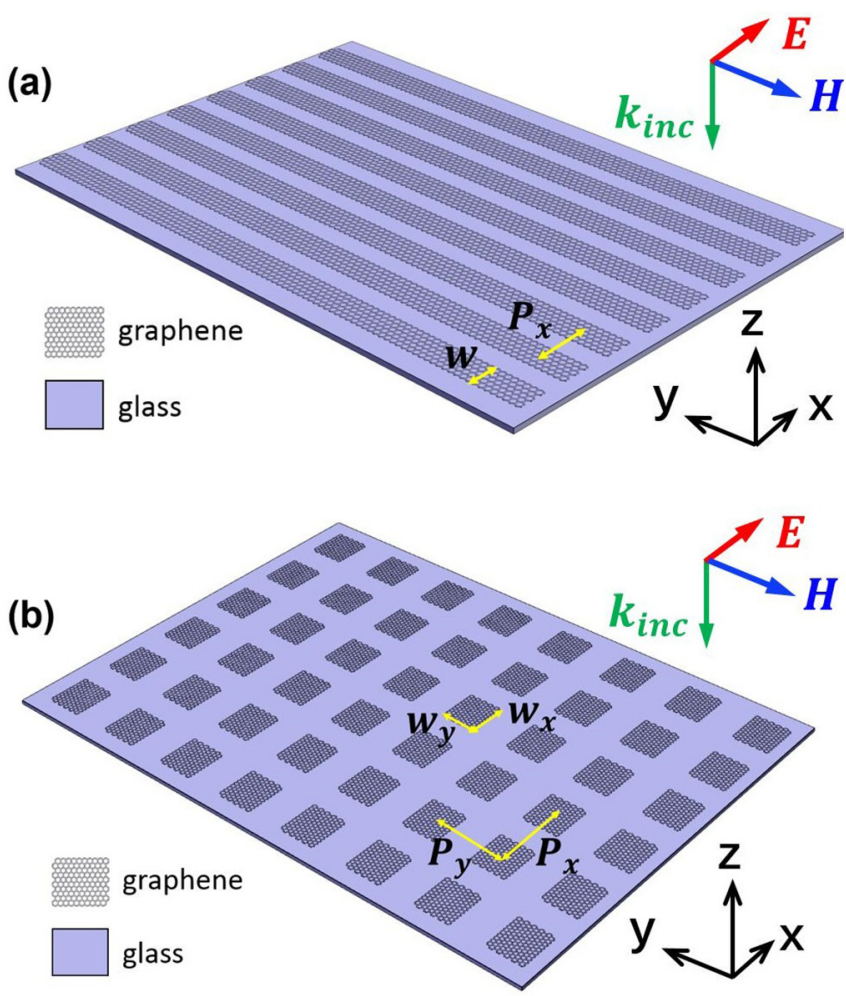

FIG. 1. (a) Schematics of a 1D graphene metasurface, with the period $P_{x}$ and width of graphene ribbons, $w$. (b) Schematics of a 2D graphene metasurface, with periods $P_{x}$ and $P_{y}$, and side-length of the graphene patches of $w_{x}$ and $w_{y}$. The two graphene metasurfaces are illuminated by an $x$-polarized plane wave normally incident onto the metasurfaces.

constants of graphene. Since graphene is a 2D semimetal, a surface optical conductivity $\sigma_{s}$ is generally used to describe its main linear physical properties at optical frequencies. Based on Kubo's formula derived within the random-phase approximation, $\sigma_{s}$ can be expressed as the sum of the intraband $\left(\sigma_{\text {intra }}\right)$ and interband $\left(\sigma_{\text {inter }}\right)$ contributions, $\sigma_{s}=\sigma_{\text {intra }}+\sigma_{\text {inter }}$. The intraband part is given by

$$
\sigma_{\text {intra }}=\frac{e^{2} k_{B} T_{\tau}}{\pi \hbar^{2}(1-i \pi \tau)}\left[\frac{\mu_{c}}{k_{B} T}+2 \ln \left(e^{-\frac{\mu_{c}}{k_{B} T}}+1\right)\right],
$$

where $\mu_{c}$ is the chemical potential, $\tau$ is the relaxation time, $T$ is the temperature, $e$ is the electron charge, $k_{B}$ is the Boltzmann constant, and $\hbar$ is the reduced Planck's constant Throughout our analysis, we use $\mu_{c}=0.6 \mathrm{eV}, \tau=0.25 \mathrm{ps}$, and $T=300 \mathrm{~K}$. Moreover, if $\mu_{c} \gg k_{B} T$, which usually holds at room temperature, the interband part can be approximated as

$$
\sigma_{\text {inter }}=\frac{i e^{2}}{4 \pi \hbar} \ln \left[\frac{2\left|\mu_{c}\right|-\left(\omega+i \tau^{-1}\right) \hbar}{2\left|\mu_{c}\right|+\left(\omega+i \tau^{-1}\right) \hbar}\right] .
$$

If we assume that the effective thickness of graphene is $h_{\text {eff }}$, the relative electric permittivity can be calculated from the conductivity through the relation

$$
\epsilon_{\mathrm{gr}}(\omega)=1+\frac{i \sigma_{s}}{\epsilon_{0} \omega h_{\mathrm{eff}}} .
$$

The relative electric permittivity of graphene is depicted in Fig. 2, where $h_{\text {eff }}=0.3 \mathrm{~nm}$ has been used. 


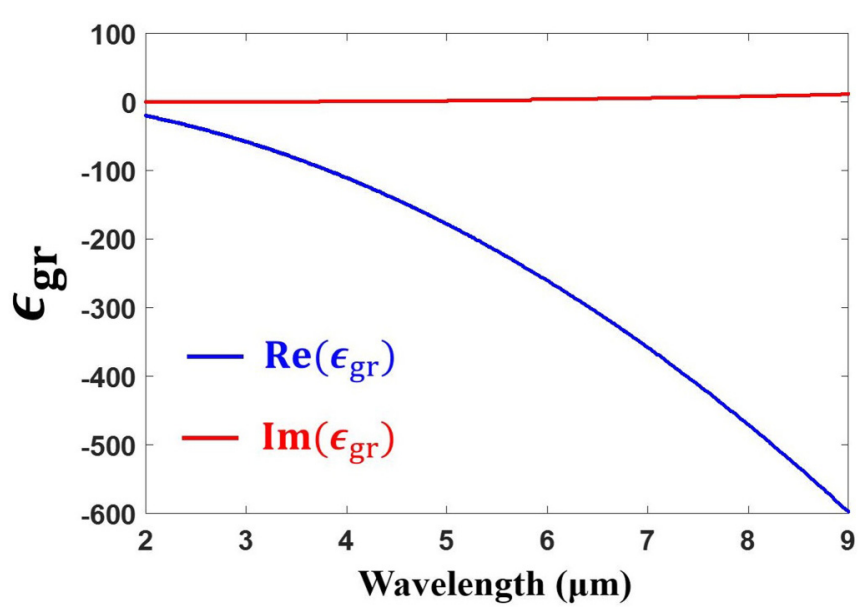

FIG. 2. Relative electric permittivity of a graphene sheet with $h_{\text {eff }}=0.3 \mathrm{~nm}$.

Similar to the case of three-dimensional (3D) bulk optical media, the nonlinear optical properties of $2 \mathrm{D}$ materials are generally determined by the symmetry properties of their atomic lattice and are quantified by (bulk) nonlinear susceptibility tensors, $\chi^{(n)}(\Omega ; \omega)$, where $\omega$ and $\Omega$ are the frequencies at the FF and higher-harmonic, respectively, and $n$ is the order of the nonlinear optical process, or, equivalently, by surface nonlinear optical conductivities, $\sigma_{s}^{(n)}(\Omega ; \omega)$. These two physical quantities are related via the following relation:

$$
\chi^{(n)}(\Omega ; \omega)=\frac{i}{\epsilon_{0} \Omega h_{\mathrm{eff}}} \sigma_{s}^{(n)}(\Omega ; \omega) .
$$

Free-standing graphene is a centrosymmetric material and therefore second-order nonlinear optical processes and, in particular, SHG are forbidden. If a graphene sheet, however, is placed onto a homogeneous substrate the inversion symmetry is broken and (dipole) SHG is allowed. In particular, such an optical configuration is characterized by a surface second-order nonlinear optical conductivity tensor, $\sigma_{s}^{(2)}(\Omega ; \omega)$, where $\Omega=2 \omega$. Symmetry considerations based on the fact that graphene belongs to the $\mathcal{D}_{6 \mathrm{~h}}$ symmetry group lead to the conclusion that this tensor has three independent nonzero components, $\sigma_{s, \perp \perp \perp}^{(2)}, \sigma_{s,\|\| \perp}^{(2)}=$ $\sigma_{s,\|\perp\|}^{(2)}$, and $\sigma_{s, \perp\|\|}^{(2)}$, where the symbols $\perp$ and $\|$ refer to the directions perpendicular to and parallel to the plane of graphene, respectively. The values of these parameters used in this paper are $\sigma_{s, \perp \perp \perp}^{(2)}=-9.71 i \times 10^{-16} \mathrm{~A} \mathrm{~m} \mathrm{~V}^{-2}, \sigma_{s,\|\| \perp}^{(2)}=$ $\sigma_{s,\|\perp\|}^{(2)}=-2.56 i \times 10^{-16} \mathrm{~A} \mathrm{~m} \mathrm{~V}^{-2}$, and $\sigma_{s, \perp\|\|}^{(2)}=-2.09 i \times$ $10^{-16} \mathrm{~A} \mathrm{~m} \mathrm{~V}^{-2},[45,46]$ and correspond to graphene placed on a silica substrate. Note that similar to the case of surface nonlinear second-order susceptibility of noble metals, the dominant component of the surface nonlinear secondorder conductivity (susceptibility) is the $\sigma_{s, \perp \perp \perp}^{(2)}\left(\chi_{s, \perp \perp \perp}^{(2)}\right)$ component.

\section{THEORY OF LINEAR AND NONLINEAR HOMOGENIZATION}

In this section, we describe a theoretical method we recently introduced [47] for the homogenization of the linear and nonlinear optical response of graphene metasurfaces. In particular, we present an approach for extracting the effective linear and nonlinear optical coefficients of a homogenized layer of material, which in the far-field has the same linear and nonlinear optical response as that of the graphene metasurface. To be more specific, we use this method to compute the effective electric permittivity of the two generic graphene metasurfaces, as well as the effective surface second-order susceptibility of graphene metasurfaces, when they are optimized to achieved maximum nonlinearity enhancement. Note that although the homogenized metasurfaces can be characterized by effective surface quantities, such as linear and nonlinear surface conductivities [48,49], in this work we consider that the homogenized metasurfaces have a finite thickness, $h_{\text {eff }}$, and thus are described by bulk effective permittivities and nonlinear susceptibilities.

To develop a general homogenization method, we extend the traditional field-averaged method to include nonlinear optical effects and anisotropic 2D materials. Thus, the constitutive relation of a linear anisotropic material is expressed as

$$
D_{i}=\sum_{j} \epsilon_{i j} E_{j},
$$

where $\mathbf{D}$ and $\mathbf{E}$ are the electric displacement and electric field, respectively, and the subscripts $i, j=x, y, z$. Then, we introduce the averaged fields, defined as

$$
\begin{aligned}
& \overline{\mathbf{D}}_{\text {eff }}(\omega)=\frac{1}{V} \int_{V} \mathbf{D}(\mathbf{r}, \omega) d \mathbf{r}, \\
& \overline{\mathbf{E}}_{\mathrm{eff}}(\omega)=\frac{1}{V} \int_{V} \mathbf{E}(\mathbf{r}, \omega) d \mathbf{r},
\end{aligned}
$$

where $V$ is the volume of the unit cell of the (1D or 2D) metasurface. More specifically, the integration domains for the $1 \mathrm{D}$ and $2 \mathrm{D}$ metasurfaces are $V=\left[0, P_{x}\right] \times\left[0, h_{\mathrm{eff}}\right]$ and $V=\left[0, P_{x}\right] \times\left[0, P_{y}\right] \times\left[0, h_{\mathrm{eff}}\right]$, respectively. Using Eqs. (5) and (6), the effective electric permittivity tensor of the metasurface, defined by the constitutive relation $\bar{D}_{i, \text { eff }}=$ $\sum_{j} \bar{\epsilon}_{i j, \text { eff }} \bar{E}_{j, \text { eff }}$, can be written as

$$
\overline{\boldsymbol{\epsilon}}_{i j \mathrm{eff}}(\omega)=\frac{\int_{V} \mathbf{D}_{i}(\mathbf{r}, \omega) d \mathbf{r}}{\int_{V} \mathbf{E}_{j}(\mathbf{r}, \omega) d \mathbf{r}}=\frac{\int_{V} \epsilon(\mathbf{r}) \mathbf{E}_{i}(\mathbf{r}, \omega) d \mathbf{r}}{\int_{V} \mathbf{E}_{j}(\mathbf{r}, \omega) d \mathbf{r}},
$$

where $\epsilon(\mathbf{r})=\epsilon_{0}$ if $\mathbf{r}$ is in air and $\epsilon(\mathbf{r})=\epsilon_{\mathrm{gr}}$ if $\mathbf{r}$ is in graphene. The formula above has been derived for metasurfaces made of isotropic optical materials, but it can be easily extended to anisotropic ones.

To assess the validity of our homogenization method, we have calculated the effective permittivity given by Eq. (7) and then compared the optical response of the homogenized metasurfaces, i.e., the absorption $A$, transmittance $T$, and reflectance $R$, with that of the two graphene metasurfaces. The optical near-fields needed to calculate $\overline{\boldsymbol{\epsilon}}_{i j \text {, eff }}(\omega)$, as well as the absorption, transmittance, and reflectance of the two graphene metasurfaces, were computed using an in-house developed code [50,51].

The effective permittivities of the homogenized metasurfaces, $\epsilon_{x x \text {,eff }}(\omega)$, retrieved using the algorithm just described, 

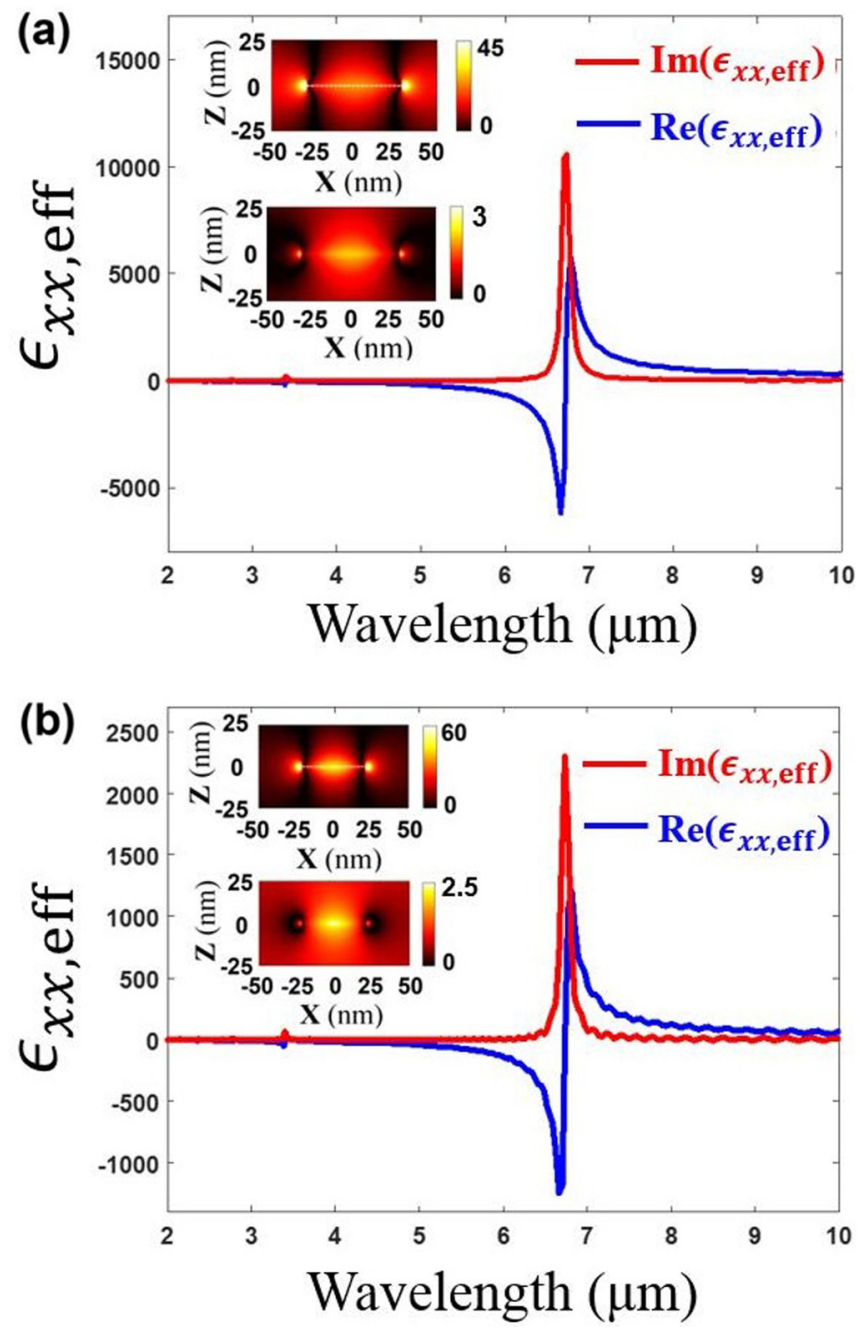

FIG. 3. (a) Effective relative permittivity of homogenized graphene-nanoribbon metasurface with $w=57.5 \mathrm{~nm}$. In insets, the spatial profile of $\left|E_{x}\right|$, calculated at the resonance wavelength $\lambda=$ $6.74 \mu \mathrm{m}$ (top panel) and at $\lambda=4 \mu \mathrm{m}$ (bottom panel). (b) The same as in (a), but calculated for the $2 \mathrm{D}$ graphene metasurface with $w_{x}=$ $42.5 \mathrm{~nm}$. The resonance wavelength for the $2 \mathrm{D}$ graphene metasurface is $\lambda=6.93 \mu \mathrm{m}$.

are presented in Fig. 3. The 1D and 2D metasurfaces considered here were optimized for maximum nonlinear response using an approach that will be described in the next section, the corresponding values of the geometrical parameters being $w=57.5 \mathrm{~nm}$ and $w_{x}=42.5 \mathrm{~nm}$, respectively. In contrast to the intrinsic permittivity of a homogeneous graphene sheet shown in Fig. 2, the effective permittivities of the homogenized metasurfaces exhibit an evident Lorentzian resonant response around a wavelength of about $6.8 \mu \mathrm{m}$, which is reminiscent of the linear optical response of an optical medium containing Lorentz-type resonators.

The field profiles presented in the insets of Figs. 3(a) and 3(b) suggest that at resonance the optical near-field is strongly enhanced, which is one of the main physical properties of SPPs. Moreover, Fig. 3 shows that in addition to this main resonance, a few other higher-order resonances exist at smaller wavelengths. These higher-order resonances correspond to the excitation of higher-order plasmon modes in
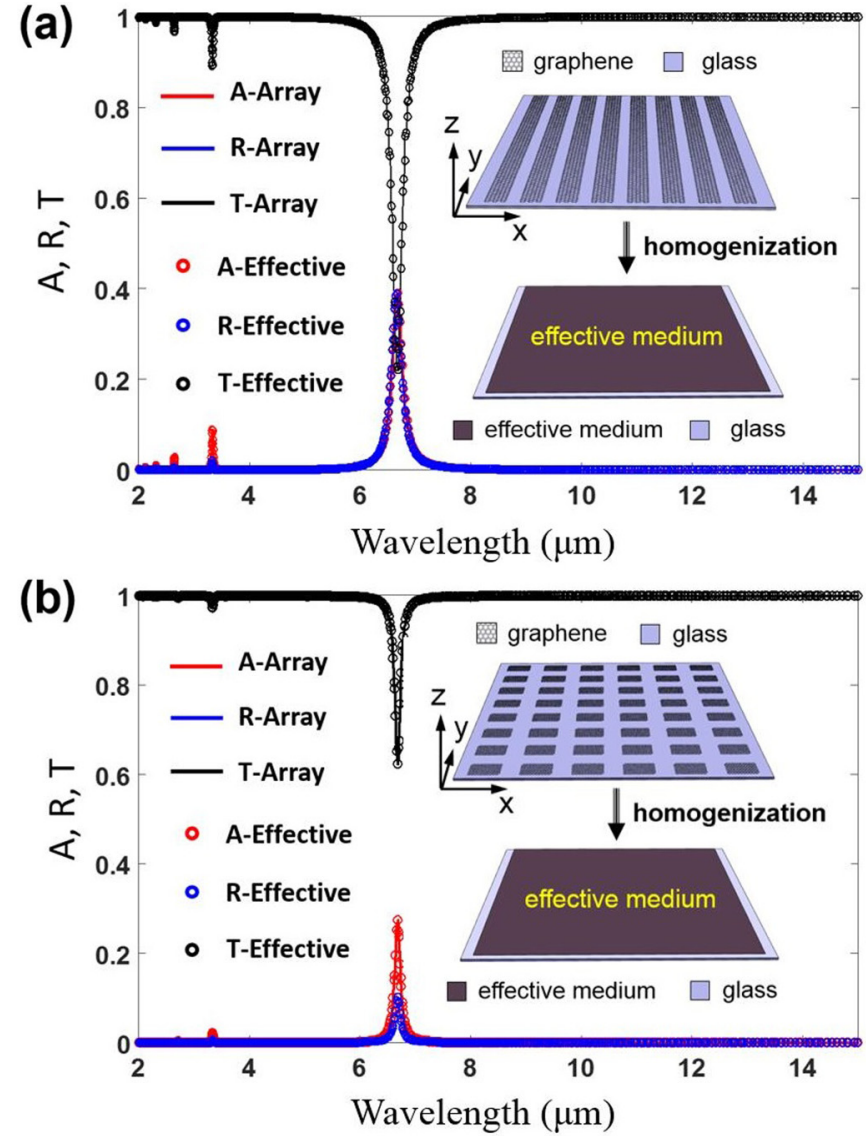

FIG. 4. Linear response comparison of absorption $A$, reflectance $R$, and transmittance $T$, calculated for the two graphene metasurfaces whose effective permittivities are presented in Fig. 3 (depicted with solid curves) and $A, R$, and $T$ corresponding to their homogenized counterparts (depicted with dotted curves).

the graphene nanoribbons or graphene patches. Interestingly enough, although graphene has metallic characteristics in the frequency range considered in our calculations, near the resonance, $\operatorname{Re}\left(\overline{\boldsymbol{\epsilon}}_{x x, \text { eff }}\right)>0$, which means that the homogenized metasurfaces behave as a dielectric around this frequency.

The main aim of a homogenization theory is to reduce a patterned metasurface to a homogeneous sheet characterized by certain effective optical constants. A reliable way to assess the validity of this procedure is to compare the optical response of the homogenized metasurface and the original one, as quantified by physical quantities such as absorption, reflectance, and transmittance. We performed this analysis for the two graphene metasurfaces whose effective permittivities are presented in Fig. 3, the corresponding results being summarized in Fig. 4. This comparison clearly demonstrates that the linear response of the homogenized sheets perfectly agrees with that of the original graphene metasurfaces, thus proving the accuracy of the proposed linear homogenization approach. This is explained by the fact that the wavelengths considered in our computations, including those at which the graphene metasurfaces are strongly resonant, are much larger than the characteristic size of the graphene constituents of the metasurfaces, so that the two optical structures are operated deep in the metasurface regime. 
We now extend the homogenization method to the nonlinear regime and use SHG as an illustrative nonlinear optical process. Thus, this nonlinear optical interaction is determined by the following nonlinear polarization:

$$
\mathbf{P}(\Omega ; \mathbf{r})=\epsilon_{0} \chi^{(2)}(\Omega ; \mathbf{r}): \mathbf{E}(\omega ; \mathbf{r}) \mathbf{E}(\omega ; \mathbf{r}),
$$

where $\Omega=2 \omega$ and $\chi^{(2)}(\Omega ; \mathbf{r})=\chi_{\mathrm{gr}}^{(2)}(\Omega)$ if $\mathbf{r}$ is in graphene and $\chi^{(2)}(\Omega ; \mathbf{r})=0$ if $\mathbf{r}$ is in air. Based on Eq. (8), the components of the SH polarization can be evaluated as

$$
P_{i}=\epsilon_{0} \sum_{j k} \chi_{i j k}^{(2)} E_{j} E_{k} \equiv \sum_{j k} q_{i j k},
$$

where we have introduced the auxiliary quantities $q_{i j k}=\epsilon_{0} \chi_{i j k}^{(2)} E_{j} E_{k}$. The averaged value of these auxiliary quantities are

$$
\bar{q}_{i j k}(\Omega)=\frac{1}{V} \int \chi_{i j k}^{(2)}(\Omega ; \mathbf{r}) E_{j}(\omega ; \mathbf{r}) E_{k}(\omega ; \mathbf{r}) d \mathbf{r} .
$$

Similarly to Eq. (8), the nonlinear SH polarization in the homogenized metasurfaces can be written as

$$
\overline{\mathbf{P}}_{\text {eff }}(\Omega)=\epsilon_{0} \chi_{\text {eff }}^{(2)}(\Omega): \overline{\mathbf{E}}_{\text {eff }}(\omega) \overline{\mathbf{E}}_{\text {eff }}(\omega),
$$

where $\chi_{\text {eff }}^{(2)}(\Omega)$ is the effective second-order susceptibility of the homogenized metasurface.

The homogenized metasurface and the original one will have the same nonlinear optical response in the far-field if the averaged nonlinear polarization in Eq. (8) is termwise equal to the effective nonlinear polarization described by Eq. (11). Using this condition, the effective second-order susceptibility of the homogenized metasurface can be evaluated as

$$
\chi_{\mathrm{eff}, i j k}^{(2)}(\Omega)=\frac{\bar{q}_{i j k}(\Omega)}{\bar{E}_{\mathrm{eff}, j}(\omega) \bar{E}_{\mathrm{eff}, k}(\omega)} .
$$

\section{RESULTS AND DISCUSSION}

In this section, we describe our approach to optimizing the nonlinear optical response of graphene metasurfaces and quantify the nonlinearity enhancement of the optimized metasurfaces. In particular, we calculate the effective second-order susceptibility of the graphene metasurfaces and compare it to the second-order susceptibility of a graphene sheet placed onto the same silica substrate.

\section{A. Linear optical response of 1D and 2D graphene metasurfaces}

One effective approach to achieving a significant enhancement of the SHG in graphene metasurfaces is to engineer their geometrical parameters so that plasmons exist at both the FF and SH. Under these conditions, the incoming light would in-couple effectively into the metasurface, as plasmons exist at the FF, which would lead to a strong enhancement of the optical near-field at the FF, and, as per Eq. (8), of the nonlinear polarization. Moreover, if plasmons exist at the $\mathrm{SH}$, too, the nonlinear sources will radiate efficiently into the continuum, the graphene metasurface behaving in these conditions as an efficient nanoantenna.

One particularly useful tool for optimizing the linear and nonlinear optical response of graphene metasurfaces is the dispersion map of the absorption, namely, the dependence
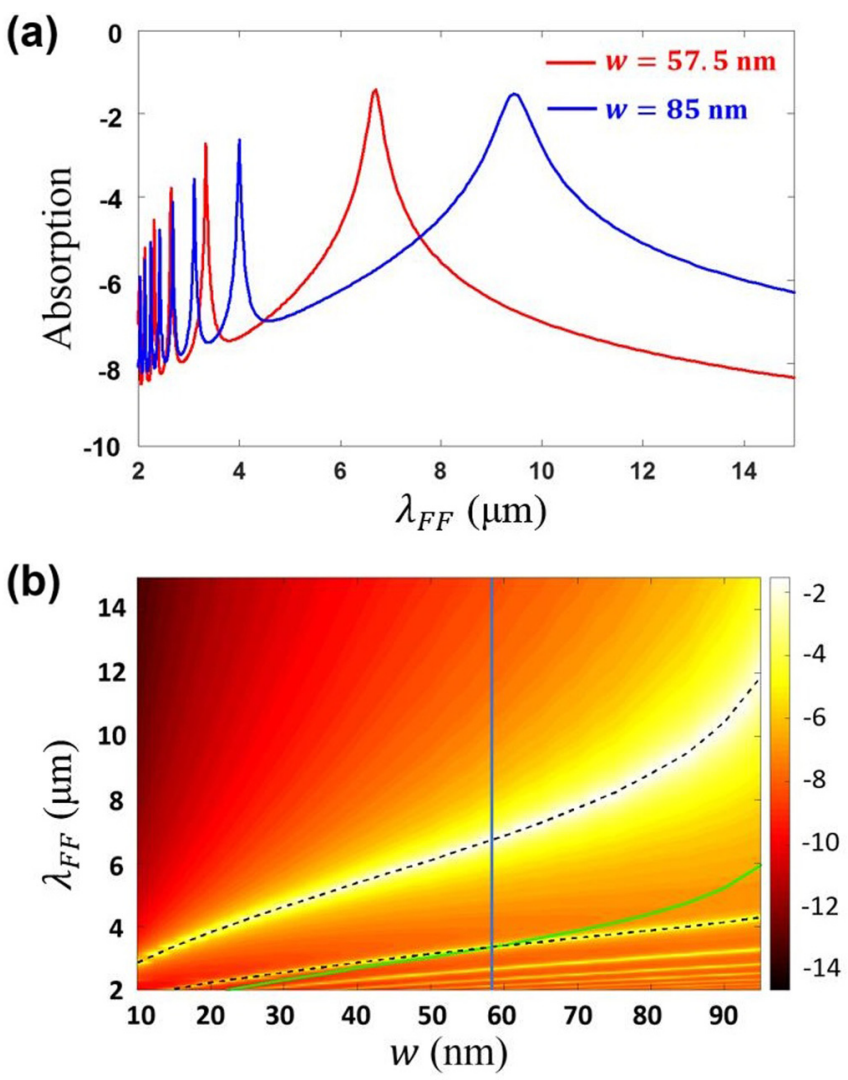

FIG. 5. (a) Absorption spectra of the 1D graphene metasurface presented in Fig. 1(a), calculated for the optimum width, $w=$ $57.5 \mathrm{~nm}$, for which a double-resonance phenomenon occurs, and for $w=85 \mathrm{~nm}$. (b) Dispersion map of absorption. Dashed curves indicate the plasmon bands, whereas the green curve indicates the half-wavelength of the fundamental plasmon band. The vertical line indicates that there is a double-resonance effect for $w=57.5 \mathrm{~nm}$.

of the optical absorption spectra on a certain parameter. Because the optical absorption increases when plasmons are excited in the structure, the absorption dispersion map provides valuable information about the frequency dispersion of the plasmon modes. The corresponding absorption spectra have been calculated using a computational method [50,51] that rigorously incorporates both the frequency dispersion and nonlinearity of graphene.

We begin our analysis with the 1D graphene metasurface presented in Fig. 1(a). Thus, we show in Fig. 5(a) the linear absorption spectra determined for the optimum width of the graphene nanoribbons, $w=57.5 \mathrm{~nm}$ (we will explain later how this value was determined) and for some other arbitrary value, $w=85 \mathrm{~nm}$. Moreover, the dispersion map of the optical absorption corresponding to this metasurface is plotted in Fig. 5(b). It can be seen in Fig. 5(a) that the absorption spectra present a series of plasmon resonances, whose amplitude decreases as the resonance wavelength decreases. These resonances appear in the absorption map as a series of geometry-dependent plasmon bands, indicated with dashed curves, with the resonance wavelength increasing with the increase of the width of the nanoribbons. Importantly, Fig. 5(b) suggests that for $w=57.5 \mathrm{~nm}$ the nanoribbons support a (fundamental) plasmon at the FF and a 

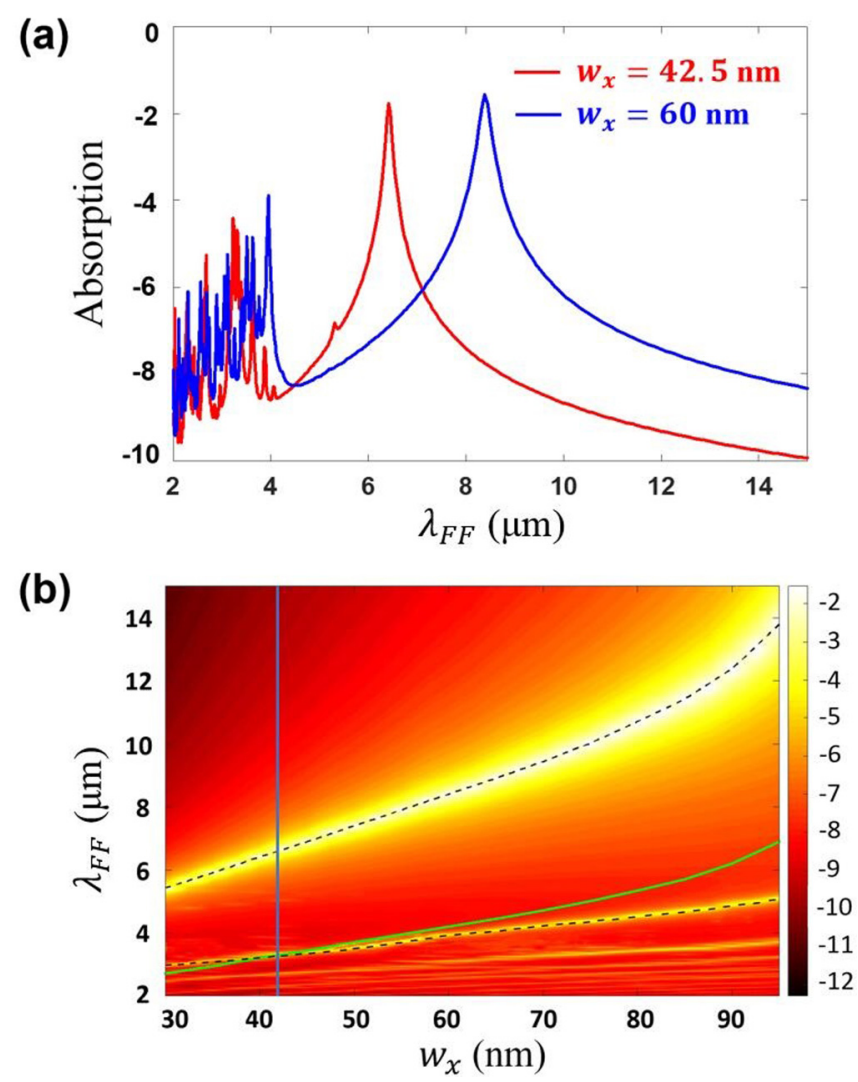

FIG. 6. (a) Absorption spectra of the 2D graphene metasurface presented in Fig. 1(b), calculated for the optimum side-length, $w_{x}=42.5 \mathrm{~nm}$, for which a double-resonance phenomenon occurs, and for $w_{x}=60 \mathrm{~nm}$. (b) Dispersion map of absorption. Dashed curves indicate the plasmon bands, whereas the green curve indicates the half-wavelength of the fundamental plasmon band. The vertical line shows that there is a double-resonance effect for $w_{x}=42.5 \mathrm{~nm}$.

second-order plasmon at the $\mathrm{SH}$, namely, the metasurface possesses a double-resonance feature.

Similar conclusions can be drawn in the case of the 2D graphene metasurface. Thus, similar to the data summarized in Fig. 5, we present in Figs. 6(a) and 6(b) two linear absorption spectra determined for the optimum side-length of the graphene patches, $w_{x}=42.5 \mathrm{~nm}$, and for an arbitrary value, $w_{x}=60 \mathrm{~nm}$, as well as the corresponding dispersion map of the optical absorption, respectively. It can be seen that in the $2 \mathrm{D}$ case, too, the resonance wavelength of the plasmon bands increases with $w_{x}$ and that the double-resonance phenomenon also occurs in 2D graphene metasurfaces. To be more specific, if $w_{x}=42.5 \mathrm{~nm}$, plasmon resonances exit at both the FF of $\lambda_{\mathrm{FF}}=6.93 \mu \mathrm{m}$, which is a fundamental plasmon, and at the $\mathrm{SH}$ of $\lambda_{\mathrm{SH}}=\lambda_{\mathrm{FF}} / 2=3.47 \mu \mathrm{m}$. Note that, as illustrated in Fig. 1(b), the 2D graphene metasurface is normally illuminated by an $x$-polarized plane wave.

The 2D graphene metasurface is anisotropic and therefore the optical absorption spectra depend on the polarization of the incident light. This idea is validated by the dispersion map of the optical absorption shown in Fig. 7, which has been determined for a normally incident, $y$-polarized incident plane wave. Thus, for this wave polarization the wavelength of fundamental-plasmon band increases with $w_{x}$, whereas

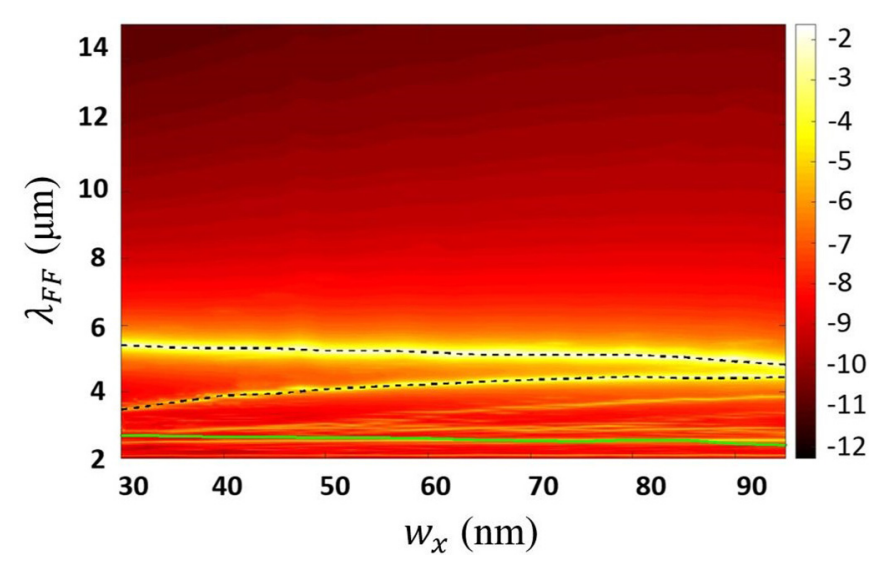

FIG. 7. The same as in Fig. 6(b) but determined for an $y$ polarized incident plane wave.

the wavelength of the higher-order plasmon bands decrease with $w_{x}$.

It can also be seen that when $w_{x}$ varies, the plasmon bands are more dispersive for $x$-polarized incident waves than for $y$ polarized waves. This finding is explained by the fact that the wavelength of the plasmon resonance is primarily determined by the size of the patch along the direction of the electric field. More importantly, however, the results in Fig. 7 suggest that the double-resonance effect does not occur for $y$-polarized incident plane waves. In our analysis, we have only considered $x$ - and $y$-polarized incident plane waves, chiefly because the conclusions for other polarizations can be derived from the results corresponding to the linear superposition of these two primary polarizations.

\section{B. Nonlinear optical response of $1 D$ and 2D graphene metasurfaces}

We now turn our attention to SHG in 1D and 2D graphene metasurfaces and investigate the influence of plasmon excitation at the FF and $\mathrm{SH}$ on the nonlinear optical response of the two graphene metasurfaces. To this end, we used a generalized-source finite-difference time-domain numerical method [51] to rigorously compute the SHG in the graphene metasurfaces. Since we want to compare the SHG intensity corresponding to different values of the width of the nanoribbons and rectangular patches, we normalize the SHG intensity to the area of the graphene structure contained in a unit cell (note that the periods $P_{x}$ and $P_{y}$ are not changed, so the areas of the unit cells do not vary). More specifically, the normalized SHG intensity spectra $I_{\mathrm{SHG}}$ were calculated as follows: In the 1D case we computed the SHG power per unit length and then divided the result by the corresponding area of the graphene nanoribbon. In the 2D case, we computed the SHG power corresponding to the unit cell with area $P_{x} \times P_{y}$ and divided the result by the area of the graphene patch, $w_{x} \times w_{y}$. Note that the normalized SHG intensity represents the sum of the SHG signals emitted in the transmission and reflection directions.

The results of these calculations are presented in Figs. 8 and 9 and correspond to the 1D and 2D metasurfaces, respectively. As Eq. (8) shows, the nonlinear polarization is proportional to the square of the optical near-field at the FF 

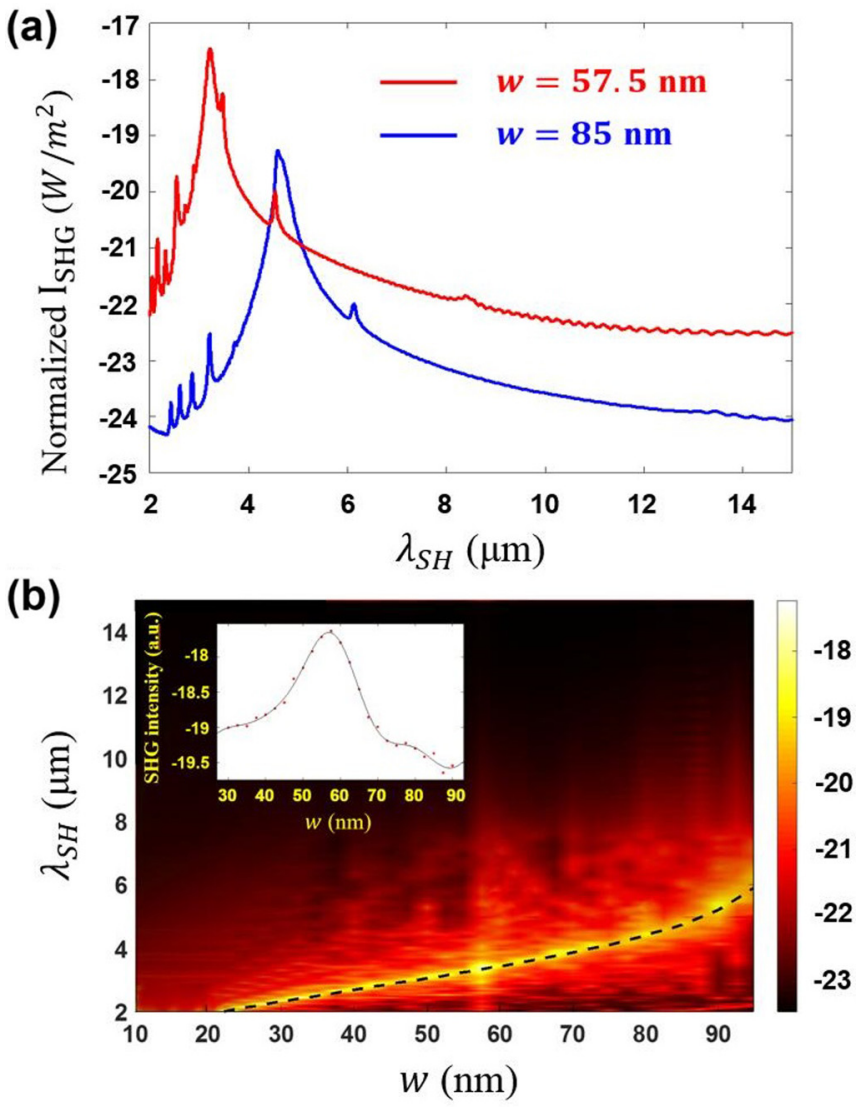

FIG. 8. (a) Normalized SHG intensity spectra $I_{\text {SHG }}$ of the $1 \mathrm{D}$ graphene metasurface presented in Fig. 1(a), calculated for the optimum width, $w=57.5 \mathrm{~nm}$, and for $w=85 \mathrm{~nm}$. (b) Dispersion map of $I_{\mathrm{SHG}}$. The dashed curve indicates the fundamental-plasmon band. The inset shows the dependence of $I_{\mathrm{SHG}}$ vs $w$, determined for the case when the wavelengths of the FF and fundamental plasmon are the same.

and therefore the SHG intensity is proportional to the FF field amplitude to the fourth. As a result, the resonance peaks of normalized SHG intensity spectra and the plasmon bands of the corresponding dispersion maps of the normalized SHG intensity should be observed at exactly the half-wavelength of the resonance peaks of linear optical absorption spectra and the corresponding plasmon bands of the dispersion maps of the linear optical absorption. This prediction is fully validated by a comparison between the results presented in Figs. 5 and 8 on the one hand, results that correspond to the 1D graphene metasurface, and, on the other hand, the results plotted in Figs. 6 and 9, which correspond to the 2D graphene metasurface.

Importantly, the insets in Figs. 8(b) and 9(b) demonstrate the SHG enhancement due to the double-resonance mechanism. Indeed, it can be inferred from these plots that for the 1D graphene metasurface, maximum SHG intensity is achieved for a width of the graphene nanoribbons of $w=57.5$ $\mathrm{nm}$, whereas in the case of the $2 \mathrm{D}$ graphene metasurface the optimum value of the side-length of the graphene patch that leads to maximum SHG intensity is $w_{x}=42.5 \mathrm{~nm}$. This clearly proves that in addition to plasmon-enhanced SHG, the double-resonance mechanism can be employed to achieve (a)
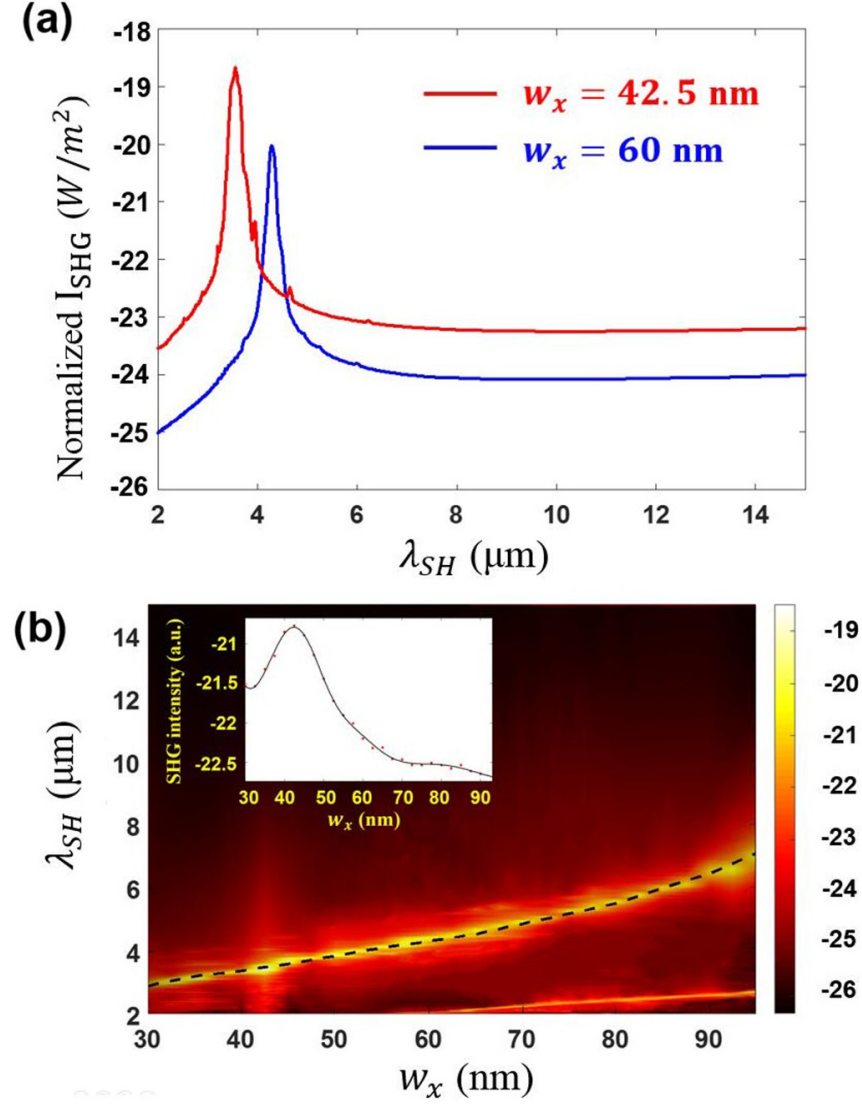

FIG. 9. (a) Normalized SHG intensity spectra $I_{\mathrm{SHG}}$ of the $2 \mathrm{D}$ graphene metasurface presented in Fig. 1(b), calculated for the optimum side-length, $w_{x}=42.5 \mathrm{~nm}$ and for $w_{x}=60 \mathrm{~nm}$. (b) Dispersion map of $I_{\mathrm{SHG}}$. Dashed curve indicates the fundamental-plasmon band. The inset shows the variation of $I_{\mathrm{SHG}}$ with $w_{x}$, computed for the case when the wavelengths of the FF and fundamental plasmon are the same.

further significant enhancement of the nonlinear optical response of graphene metasurfaces.

\section{Enhancement of the effective second-harmonic susceptibility of $1 D$ and 2D graphene metasurfaces}

A suitable physical quantity that measures the enhancement of the nonlinear optical response of a nonlinear optical system is the nonlinear susceptibility. Therefore, we have used the homogenization method described in Sec. III to calculate the effective second-order susceptibility of the two graphene metasurfaces. In particular, we retrieved the three independent components of this nonlinear susceptibility, $\chi_{\mathrm{eff}, z z z}^{(2)}, \chi_{\mathrm{eff}, x x z}^{(2)}$, and $\chi_{\mathrm{eff}, z x x}^{(2)}$. The results of these calculations are summarized in Figs. 10 and 11, and correspond to the 1D and 2D metasurfaces, respectively.

One important conclusion that can be inferred from the data presented in these figures is that, similar to the case of the effective permittivity of the homogenized graphene metasurfaces, all components of the effective second-order susceptibilities show a resonant behavior around the plasmon resonance wavelength (fundamental and higher-order wavelength), which means that the enhancement of the 
(a)

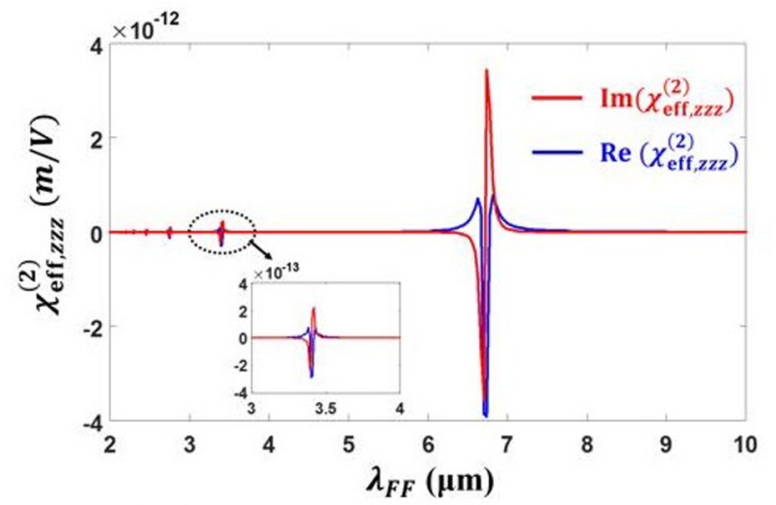

(b)

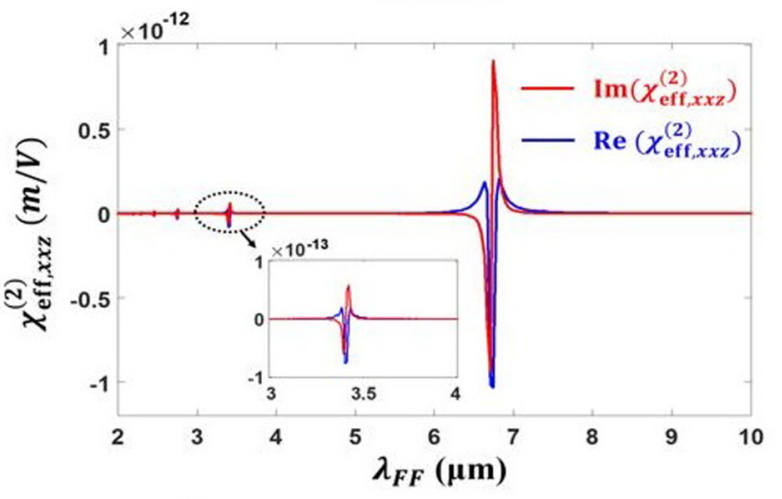

(c)

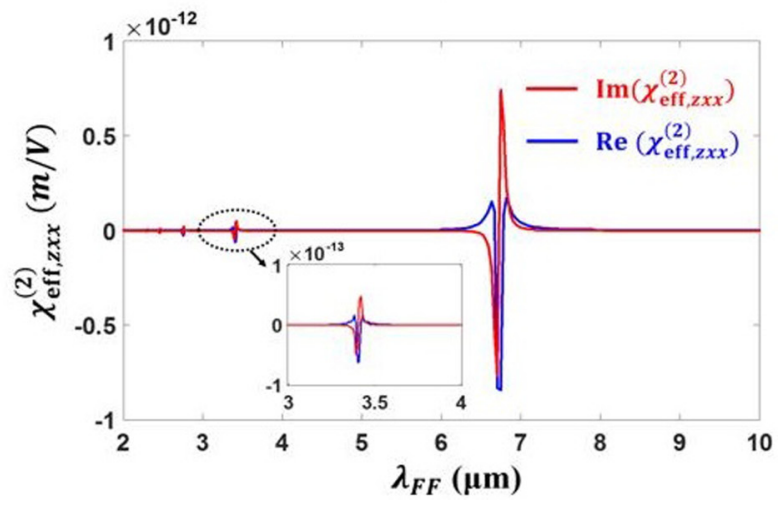

FIG. 10. Wavelength dependence of the three independent components of the effective second-order susceptibility $\chi_{\mathrm{eff}}^{(2)}$ of the 1D graphene metasurface.

nonlinearity of the graphene metasurfaces can be traced to the excitation of graphene SPPs. The maximum enhancement occurs when the fundamental plasmon is excited. Moreover, the spectra of these components of the second-order susceptibilities are similar to those of a nonlinear optical medium containing resonators of Lorentzian nature, which suggests that the graphene nanostructures that constitute the building blocks of the two metasurfaces can be viewed as meta-atoms responsible for the effective nonlinear optical response of these optical nanostructures. Since the size of these metaatoms is much smaller than the resonance wavelength at the $\mathrm{SH}$, one can conclude that the nonlinear graphene gratings investigated in this study operate in the metasurface regime, too.

To further analyze the characteristics of the magnitude of the enhancement of the nonlinear optical response of the two graphene metasurfaces, we also calculated the (a)

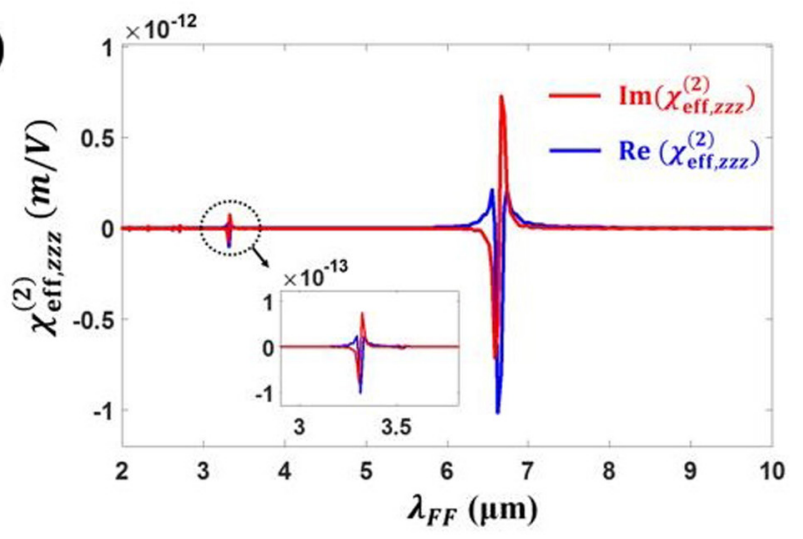

(b)

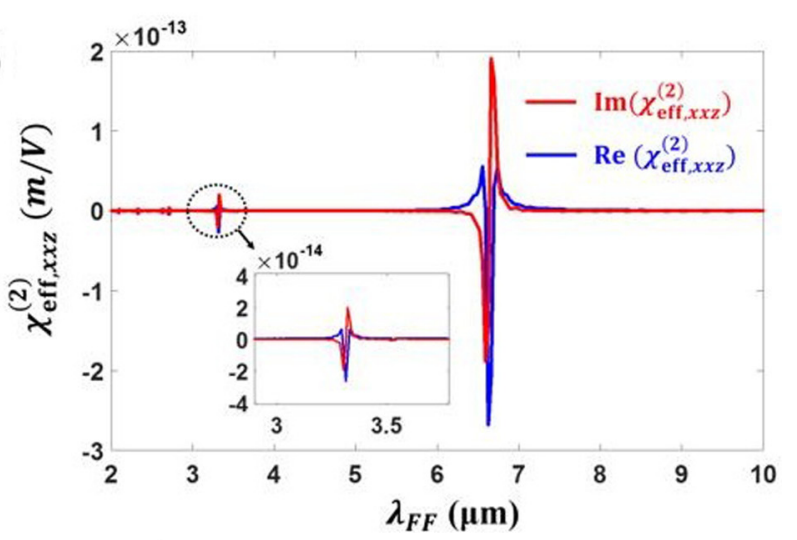

(c)

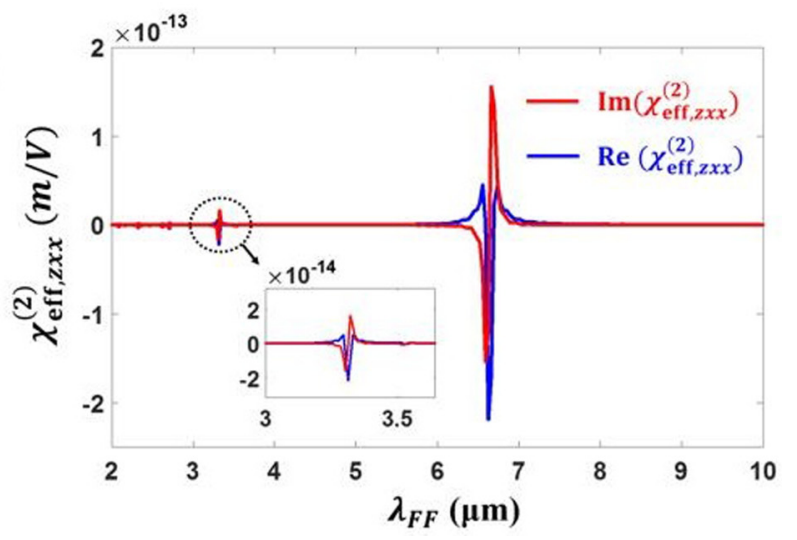

FIG. 11. The same as in Fig. 10, but corresponding to the 2D graphene metasurface.

enhancement factors $\eta_{z z z}=\left|\chi_{\mathrm{eff}, z z z}^{(2)} / \chi_{\mathrm{gr}, z z z}^{(2)}\right|, \eta_{x x z}=\left|\chi_{\mathrm{eff}, x x z}^{(2)}\right|$ $\chi_{\mathrm{gr}, x x z}^{(2)} \mid$, and $\eta_{z x x}=\left|\chi_{\mathrm{eff}, z x x}^{(2)} / \chi_{\mathrm{gr}, z x x}^{(2)}\right|$ for several different values of the angle of incidence $\theta$. In these definitions, $\chi_{\mathrm{gr}}^{(2)}$ is the surface second-order susceptibility of a uniform graphene sheet placed on top of a silica substrate.

The results of this analysis are summarized in Fig. 12, where we show the data corresponding to the enhancement $\eta_{z z z}$ of the dominant component of $\chi_{\mathrm{eff}}^{(2)}$ of the 1D and 2D metasurfaces. This figure demonstrates a remarkable enhancement of the second-order nonlinearity of the two metasurfaces, especially near the plasmon resonance. In particular, the dominant component $\chi_{\mathrm{eff}, z z z}^{(2)}$ of the homogenized graphene metasurfaces is larger by more than three orders of magnitude than the corresponding component $\chi_{\mathrm{gr}, z z z}^{(2)}$ of a graphene sheet placed on the same silica substrate. It can also be observed 

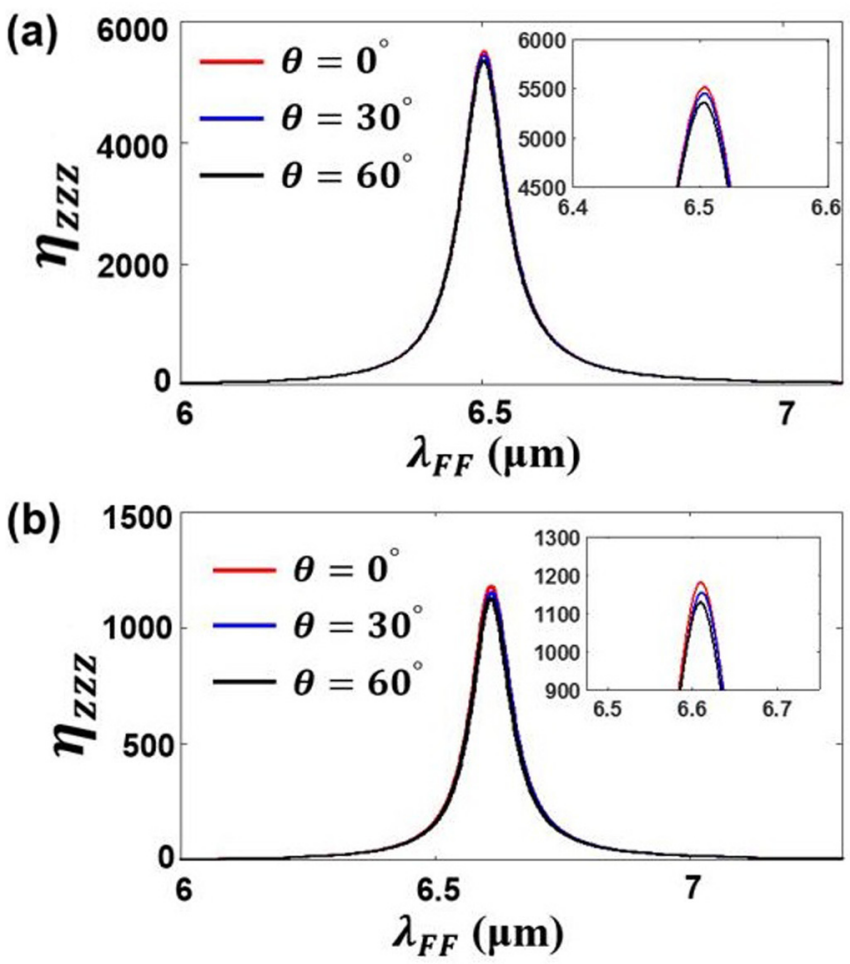

FIG. 12. (a) Wavelength dependence of the enhancement factor of the dominant component of the effective second-order susceptibility, determined for the optimized 1D graphene metasurface for several values of the angle of incidence $\theta$. (b) The same as in (a), but determined for the $2 \mathrm{D}$ optimized graphene metasurface.

that $\eta_{z z z}$ only slightly decreases as the angle of incidence increases, which further proves that the graphene elements of the metasurfaces behave as true metaatoms. We also stress that despite the fact that the nonlinear optical losses are enhanced as well around plasmon resonances, one expects that this is not a particularly detrimental effect as the graphene metasurfaces investigated in this work are not meant to be employed in applications where large propagation distances are required.

\section{CONCLUSION}

In summary, in this study we investigated the optical response of one- and two-dimensional graphene metasurfaces and their homogenized counterparts. In particular, using a recently developed homogenization technique, we retrieved the effective permittivity and effective second-order susceptibility of the homogenized metasurfaces and compared the values of several physical quantities characterizing the original and homogenized metasurfaces, such as the optical absorption, transmittance, and reflectance. Our analysis revealed that for metasurfaces whose graphene constituents have characteristic sizes of a few tens of nanometers there is an excellent agreement between the predictions of the homogenization method and the results obtained by rigorously solving the Maxwell equations. This was explained by the fact that the characteristic size of graphene resonators is much smaller than their resonance wavelength.

Our theoretical analysis of the two types of homogenized graphene metasurfaces showed that their nonlinear responses can be greatly enhanced when surface plasmons are excited in their graphene constituents. Additional nonlinearity enhancement is achieved when plasmons exist at both the fundamental frequency and second harmonic, the overall effect of this double-resonance effect being an enhancement of the effective second-order susceptibility of the graphene metasurfaces by more than three orders of magnitude. Moreover, it should be noted that this double-resonance phenomenon could also be observed in other more complex configurations, e.g., when plasmons are excited in different plasmonic materials, such as metasurfaces containing coupled metallic-graphene nanostructures. Equally important, the proposed homogenization method can be readily extended to other cases, too, such as three-dimensional configurations or incident waves with arbitrary polarization and angle of incidence, which further underscores the importance of the results reported in this study.

\section{ACKNOWLEDGMENTS}

This work was supported by the European Research Council (ERC), Grant Agreement No. ERC-2014-CoG-648328, China Scholarship Council (CSC), and University College London (UCL) (201506250086). The authors acknowledge the use of the UCL Legion High Performance Computing Facility (Legion@UCL) and associated support services in the completion of this work.
[1] A. Alu, M. G. Silveirinha, A. Salandrino, and N. Engheta, Phys. Rev. B 75, 155410 (2007).

[2] T. Cao, C. W. Wei, R. E. Simpson, L. Zhang, and M. J. Cryan, Sci. Rep. 4, 3955 (2014).

[3] J. W. You and N. C. Panoiu, Opt. Express 26, 1882 (2018).

[4] M. Veysi, C. Guclu, O. Boyraz, and F. Capolino, J. Opt. Soc. Am. B 32, 318 (2015).

[5] G. Ren, Z. Lai, C. Wang, Q. Feng, L. Liu, K. Liu, and X. Luo, Opt. Express 18, 18151 (2010).

[6] C. Ma and Z. Liu, Opt. Express 18, 4838 (2010).

[7] I. Al-Naib, G. Sharma, M. M. Dignam, H. Hafez, A. Ibrahim, D. G. Cooke, T. Ozaki, and R. Morandotti, Phys. Rev. B 88, 195203 (2013).
[8] P. Ding, E. Liang, G. Cai, W. Hu, C. Fan, and Q. Xue, J. Opt. 13, 075005 (2011).

[9] J. Zhang, W. Liu, Z. Zhu, X. Yuan, and S. Qin, Opt. Express 22, 30889 (2014).

[10] B. Kante, A. de Lustrac, and J. M. Lourtioz, Phys. Rev. B 80, 035108 (2009).

[11] A. V. Kabashin, P. Evans, S. Pastkovsky, W. Hendren, G. A. Wurtz, R. Atkinson, and A. V. Zayats, Nat. Mater. 8, 867 (2009).

[12] K. V. Sreekanth, Y. Alapan, M. ElKabbash, E. Ilker, M. Hinczewski, U. A. Gurkan, and G. Strangi, Nat. Mater. 15, 621 (2016).

[13] C. Cao, J. Zhang, X. Wen, S. L. Dodson, N. T. Dao, L. M. Wong, and Q. Xiong, ACS Nano 7, 7583 (2013). 
[14] N. Vasilantonakis, G. A. Wurtz, V. A. Podolskiy, and A. V. Zayats, Opt. Express 23, 14329 (2015).

[15] N. I. Landy, S. Sajuyigbe, J. J. Mock, D. R. Smith, and W. J. Padilla, Phys. Rev. Lett. 100, 207402 (2008).

[16] R. Alaee, M. Farhat, C. Rockstuhl, and F. Lederer, Opt. Express 20, 28017 (2012).

[17] S. Song, Q. Chen, L. Jin, and F. Sun, Nanoscale 5, 9615 (2013).

[18] D. Schurig, J. J. Mock, B. J. Justice, S. A. Cummer, J. B. Pendry, A. F. Starr, and D. R. Smith, Science 314, 977 (2006).

[19] D. Shin, Y. Urzhumov, Y. Jung, G. Kang, S. Baek, M. Choi, and D. R. Smith, Nat. Commun. 3, 1213 (2012).

[20] Z. Liu, S. Durant, H. Lee, Y. Pikus, Y. Xiong, C. Sun, and X. Zhang, Opt. Express 15, 6947 (2007).

[21] D. Korobkin, B. Neuner, C. Fietz, N. Jegenyes, G. Ferro, and G. Shvets, Opt. Express 18, 22734 (2010).

[22] S. Maslovski and S. Tretyakov, New J. Phys. 14, 035007 (2012).

[23] G. Rosenblatt and M. Orenstein, Phys. Rev. Lett. 115, 195504 (2015).

[24] J. S. Gomez-Diaz, M. Tymchenko, J. Lee, M. A. Belkin, and A. Alu, Phys. Rev. B 92, 125429 (2015).

[25] A. Rose and D. R. Smith, Opt. Mater. Express 1, 1232 (2011).

[26] C. Argyropoulos, G. D’Aguanno, and A. Alu, Phys. Rev. B 89, 235401 (2014).

[27] E. Almeida, G. Shalem, and Y. Prior, Nat. Commun. 7, 10367 (2016).

[28] G. Li, S. Zhang, and T. Zentgraf, Nat. Rev. Mater. 2, 17010 (2017).

[29] F. Qin, L. Ding, L. Zhang, F. Monticone, C. C. Chum, J. Deng, and S. Zhang, Sci. Adv. 2, e1501168 (2016).

[30] Y. Zhao and A. Alu, Phys. Rev. B 84, 205428 (2011).

[31] Y. Yang, I. I. Kravchenko, D. P. Briggs, and J. Valentine, Nat. Commun. 5, 5753 (2014).

[32] J. Sautter, I. Staude, M. Decker, E. Rusak, D. N. Neshev, I. Brener, and Y. S. Kivshar, ACS Nano 9, 4308 (2015).
[33] S. A. Maier, Plasmonics: Fundamentals and Applications (Springer, New York, 2007).

[34] A. V. Zayats, I. I. Smolyaninov, and A. A. Maradudin, Phys. Rep. 408, 131 (2005).

[35] F. J. Garcia-Vidal, L. Martin-Moreno, T. W. Ebbesen, and L. Kuipers, Rev. Mod. Phys. 82, 729 (2010).

[36] N. C. Panoiu, W. E. I. Sha, D. Y. Lei, and G. C. Li, J. Opt. 20, 083001 (2018).

[37] J. B. Khurgin, Nat. Nanotechnol. 10, 2 (2015).

[38] T. Christopoulos, O. Tsilipakos, N. Grivas, and E. E. Kriezis, Phys. Rev. E 94, 062219 (2016).

[39] T. Christopoulos, O. Tsilipakos, G. Sinatkas, and E. E. Kriezis, Phys. Rev. B 98, 235421 (2018).

[40] V. G. Ataloglou, T. Christopoulos, and E. E. Kriezis, Phys. Rev. A 97, 063836 (2018).

[41] F. H. Koppens, D. E. Chang, and F. J. Garcia de Abajo, Nano Lett. 11, 3370 (2011).

[42] S. H. Lee, M. Choi, T. T. Kim, S. Lee, M. Liu, X. Yin, and X. Zhang, Nat. Mater. 11, 936 (2012).

[43] J. W. You, S. R. Bongu, Q. Bao, and N. C. Panoiu, Nanophotonics 8, 63 (2019).

[44] J. W. You, J. You, M. Weismann, and N. C. Panoiu, Philos. Trans. R. Soc. London A 375, 20160313 (2017).

[45] Y. Q. An, J. E. Rowe, D. B. Dougherty, J. U. Lee, and A. C. Diebold, Phys. Rev. B 89, 115310 (2014).

[46] J. J. Dean and H. M. van Driel, Phys. Rev. B 82, 125411 (2010).

[47] J. W. You and N. C. Panoiu, arXiv:1904.11297.

[48] C. L. Holloway, E. F. Kuester, and A. Dienstfrey, IEEE Antenn. Wireless Propag. Lett. 10, 1507 (2011).

[49] O. Tsilipakos, T. Koschny, and C. M. Soukoulis, ACS Photon. 5, 1101 (2018).

[50] M. Weismann and N. C. Panoiu, Phys. Rev. B 94, 035435 (2016).

[51] J. W. You, E. Threlfall, D. F. Gallagher, and N. C. Panoiu, J. Opt. Soc. Am. B 35, 2754 (2018). 